\title{
Comparative Reliability Assessment of Tooth Volume Measurement with Different Three-Dimensional Imaging Software
}

\author{
Tara Ali Rasheed $\mathbb{D}^{1}{ }^{1}$ Afrah Khazal Shehab Al Hamdany, ${ }^{2}$ and Aras Maruf Rauf ${ }^{3}$ \\ ${ }^{1}$ Department of Pedodontics, Orthodontics, and Prevention, College of Dentistry, University of Sulaimani, Sulaimani-shorsh 67/4, \\ Kurdistan Region, Iraq \\ ${ }^{2}$ Department of Pedodontics, Orthodontics, and Prevention, College of Dentistry, University of Mosul, \\ Mosul-Alsidik 345/207/512, Iraq \\ ${ }^{3}$ Department of Pedodontics, Orthodontics, and Prevention, College of Dentistry, University of Sulaimani, Sulaimani- \\ Tooymalik 211/61, Kurdistan Region, Iraq
}

Correspondence should be addressed to Tara Ali Rasheed; tara.rasheed@univsul.edu.iq

Received 8 August 2020; Revised 18 October 2020; Accepted 25 October 2020; Published 7 November 2020

Academic Editor: Vincenzo Iorio Siciliano

Copyright (C) 2020 Tara Ali Rasheed et al. This is an open access article distributed under the Creative Commons Attribution License, which permits unrestricted use, distribution, and reproduction in any medium, provided the original work is properly cited.

\begin{abstract}
Objective. To evaluate the in vivo tooth volume through VRMesh and 3Matic programs and to compare the measurements to the physical volume. So, the aim of the study was to ensure the reliability and sensitivity of the three-dimensional software (VRMesh and 3Matic) in measuring tooth volume. Material and Methods. The volume of 26 extracted upper first premolars from orthodontic patients who had CBCT before orthodontic treatment were measured. Two different commercial programs, which were VRMesh and 3Matic, were used to calculate the volume of the segmented upper first premolar from CBCT. The in vivo tooth volume was compared to the physical tooth volume to examine the accuracy of the two software in measuring the tooth volume. Results. The difference between the mean of the in vivo and in vitro tooth volume measurements was too small, making it clinically nonsignificant. ANOVA test was used as a statistical tool, and no statistically significant difference was noticed among the measurements. The values were normally distributed when tested for normality by Kolmogorov-Smirnov and Shapiro-Wilk test. $\boldsymbol{P}$ value less than or equal to $0.05(\boldsymbol{P} \leq 0.05)$ was considered statistically significant. Conclusion. The assessment of the in vivo tooth volume measurement with different three-dimensional imaging software (VRMesh and 3Matic) programs in comparison with the tooth physical volume is reliable. The use of a mouse pen during the refining stage of the segmentation may have increased the accuracy of the procedure. The determined in vivo tooth volumes are dependable and can be applied in orthodontic diagnosis and treatment planning.
\end{abstract}

\section{Introduction}

Evaluation of tooth volume is of great importance in dentistry generally and as specific consideration in orthodontic treatments and biomechanics. In Europe, Cone Beam, Computed Tomography (CBCT) was introduced to dentistry in 1998 [1], while in the USA, it was approved for use in 2001 [2]. Since then, CBCT technology has gone through a drastic evolution, due to great demands from each specialty for its accurate, reproducible, and safe three-dimensional images.
In orthodontics, three-dimensional imaging raises the possibility of increasing diagnostic ability, with very practical and easy application in daily orthodontic procedures $[3,4]$. In orthodontics, generally, СBCT images are used with a small field of view (FOV) $[5,6]$ which has approximately high contrast image compared with the larger ones [7]. FOV refers to the size of the scan volume that is necessary to adequately capture the region of interest [8-10].

Developmental advancements in the use of CBCT technology and its three-dimensional imaging software have been 
seen in orthodontic practice, which has basically branched from an increased employment of the tool in this dental branch clinically. The applications of three-dimensional imaging are certainly developed with advances in CBCT technology and expanding software capability. Tooth size cannot be measured by two-dimensional images such as CBCT, while the three-dimensional imaging of tooth anatomical structure can aid in calculating the tooth volume [11].

The practicability of in vivo dental volume measurements by using CBCT imaging was reported by Liu et al. [12]. Furthermore, CBCT images are anatomically true to size, compared to conventional cephalometric radiographs [13-15]. Designation of a three-dimensional model and the virtual imaging which illustrates the whole tooth structure and the surrounding hard tissues with craniofacial structures would greatly assist the orthodontist to think about different treatment possibilities throughout the steps of diagnosis and treatment planning of a case. Additionally, by observing the changes that occur during the treatment, the final results can be estimated and predicted accurately [16]. The bracket positioning by indirect technique is another important indication of the virtual model in the orthodontic branch $[7,17]$, especially for lingual appliances [5], producing accurate wire bending, and correct surgical simulation during orthognathic surgery $[6,18]$.

Three-dimensional measurements from CBCTs can produce several visualization modes which are multiplanar (MPR), volume-rendered (VR), and shaded surface display (SSD) $[19,20]$.

Elimination of all structures surrounding an object such as a tooth in CBCT, called segmentation, is necessary during volumetric analysis and it can be done by automated, semiautomated, or manual image thresholding. The thresholding which is applied during segmentation is the process of dividing an image into many smaller images with boundaries defined by grayscale values [21-23]. Due to the presence of human bias, the image segmentation process can often be challenging [24].

According to the amount of radiation absorbed, each voxel has its own specific grayscale value. If a single voxel consists of tissues with different densities, the average of the grayscale of that voxel is taken during three-dimensional reconstruction. The grayscale values obtained from CBCT imaging cannot be used quantitatively as a result of voxel averaging $[8,25,26]$.

The volume of the tooth can also be calculated physically by the water displacement method through subtracting the initial water volume from the final volume after immersing the tooth in the water $[27,28]$.

\section{Material and Method}

2.1. The Sample. A total of 26 sound teeth (upper first premolars) from 13 Kurdish orthodontic patients who needed bilateral extraction of both the upper first premolars as a part of the orthodontic treatment plan were evaluated in this study. All the patients were 18 to 25 years of age, having CBCT taken before their orthodontic procedure.
2.2. Radiographic Imaging. A CBCT image was taken for each patient as a part of their orthodontic treatment plan with a CBCT machine (NewTom VGi scanner; QR s.r.l., Verona, Italy); the exposure was set as $90 \mathrm{kV}$ (tube voltage), $3.00 \mathrm{~mA}$ (tube current), 9.0 second exposure time, and a voxel size of $0.150 \mathrm{~mm}$ (the voxel is the minimum unit of digital data segmentation in three-dimensional space). The acquired data from the X-ray machine were exported using a specific type of file (Digital Imaging and Communication in Medicine (DICOM)).

2.3. Three-Dimensional Measurement. The volume of each tooth was calculated using two different software as follows: at the first occasion, the Digital Imaging and Communication in Medicine (DICOM) files were imported into an image processing software for three-dimensional design and modeling called Materialise Interactive Medical Image Control System (MIMICS) program which is usually used to create a three-dimensional surface model from stacks of twodimensional image data (Figure 1).

After the three-dimensional tooth model was completed, the DICOM file was sent to the 3Matic (3-Matic AnalyzeMimics Innovation Suite, Materialise, Leuven, Belgium) program from MIMICS in which the first calculation tooth volume was done (Figure 2). VRMesh (Virtual Grid, Bellevue City, WA) was another software being used in the present study to estimate the tooth volume (Figure 3); this software was developed by an American company and is used in many fields including dentistry. The VRMesh program cannot recognize DICOM files, so the 3Matic software was used in converting the files into stereolithographic (Figure 4) or Standard Tessellation Language (STL) as shown in Figures 5(a) and 5(b).

Tooth segmentation was performed on consecutive twodimensional slices by semiautomated segmentation with manual localized visual refinement on a repeated twodimensional basis as it was regarded as a reliable method for leading to an accurate approach of volume quantitative analysis in different studies $[29,30]$. A visually defined optimal threshold value was set for each tooth in the sagittal (YZ) plane. The threshold level was set individually with regard to each patient according to the density values which are called Hounsfield units (HU), and once it is determined, it would not be altered during the entire process of the $3 \mathrm{D}$ model construction. The tooth anatomy should be obvious and clear with minimal interference from the surrounding bone and adjacent structures when the threshold value was set. Manual refinements were handled slice-by-slice for more accuracy by correcting the under- and/or overcontoured voxels in the tooth volume [31].

Initial refinements were done in the $\mathrm{YZ}$ plane, and the second ones were performed in the axial (XY) plane to clarify the root structure and interproximal dental contact points, while the third refinements were completed in the $\mathrm{XZ}$ (coronal) plane to preserve tooth anatomy and focus on the delineation of dental root structure from the buccal and palatal cortical plates (Figure 1). The 3D resultant tooth was assessed for approximately normal maxillary first premolar dental anatomy. In all manual outlining and refinement steps 


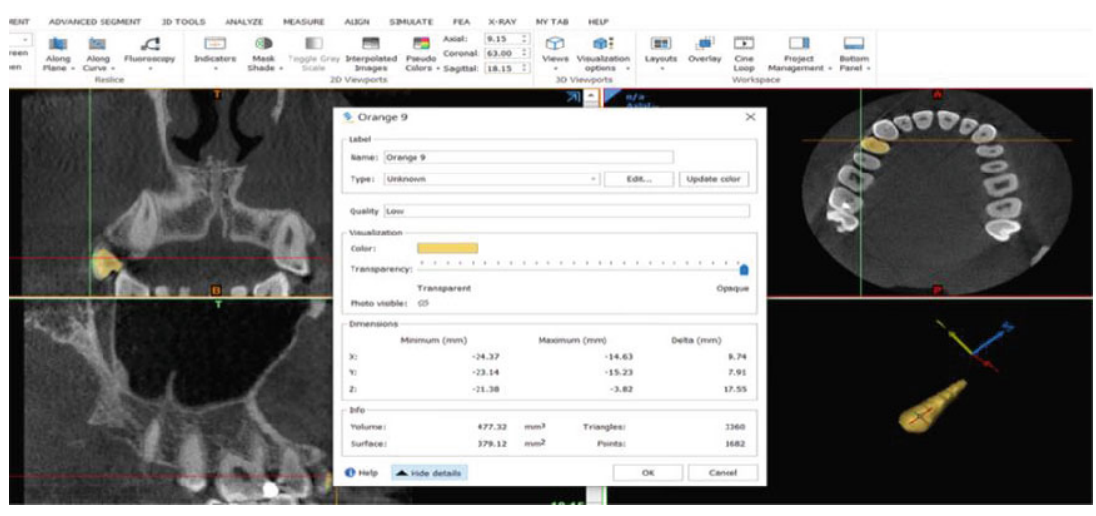

FIgURE 1: Creation of the three-dimensional tooth model by MIMICS from CBCT.

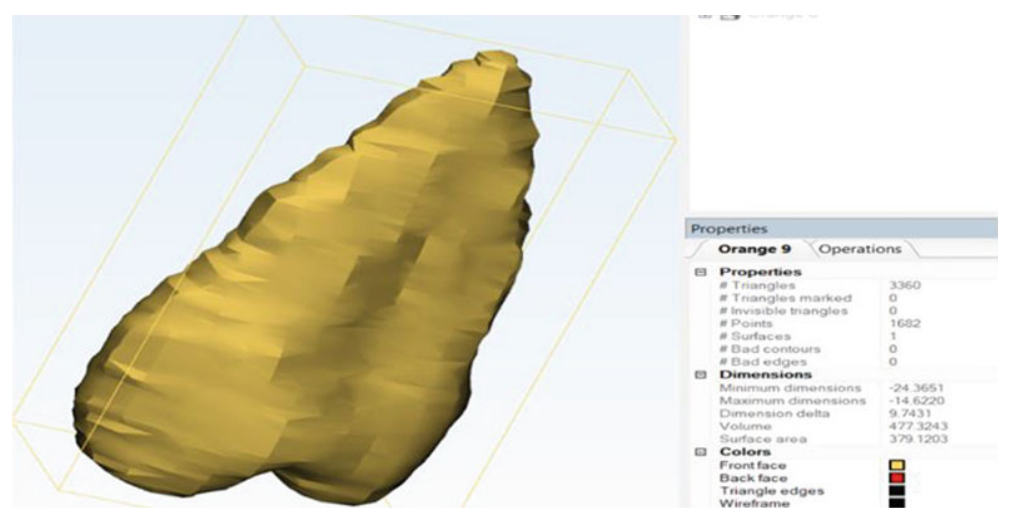

FIGURE 2: Evaluating the volume of the segmented tooth by the 3 Matic software.

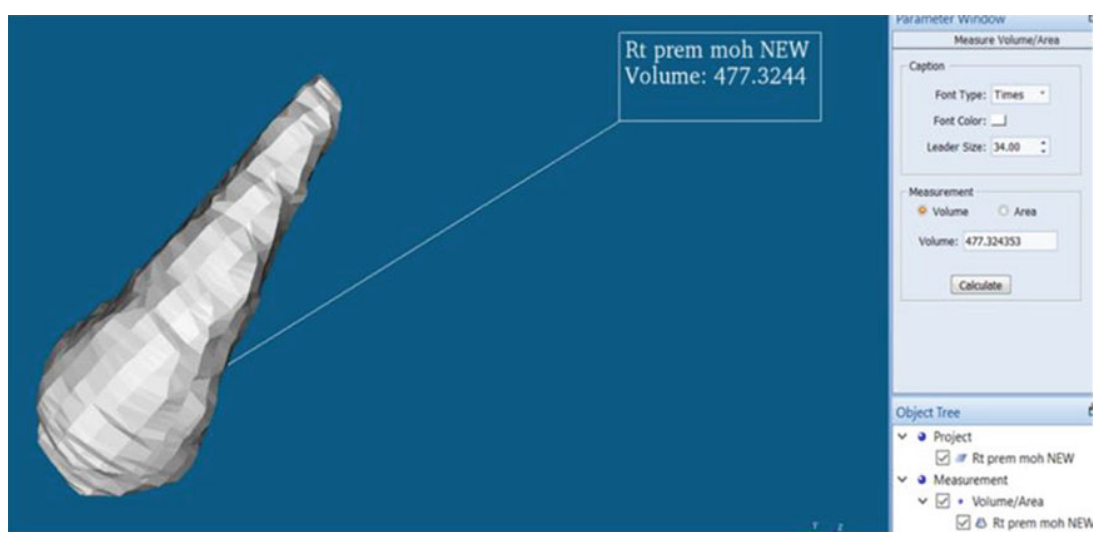

FIGURE 3: Measuring of the tooth by the VRMesh software.

of the teeth, an external mouse pen was used as a tool connected to the computer for more accurate results. No smoothing functions were applied to the three-dimensional tooth structure to prevent the flattening of minor root defects/imperfections or possible resorption lacunae [30].

The segmentation of the images was a sensitive process; to avoid any misinterpretation, the images were segmented by two observers after which an interexaminer calibration was done to avoid any kind of reading bias. Moreover, the segmentation process was repeated blindly by the researcher within two-week intervals, and they were color coded in the same DICOM volumetric data to facilitate differentiation. After segmentation, the software automatically computed each tooth's radiographic volume from the stack of segmented two-dimensional slices, and the three-dimensional shape of the tooth was prepared. The measurements obtained from the software were in cubic millimeters $\left(\mathrm{mm}^{3}\right)$ which are converted to milliliters $(\mathrm{mL})$ to unify the readings with the physical volume calculations.

2.4. Measurement of Tooth Physical Volume. In order to measure the real volume, each studied tooth was extracted, gently 


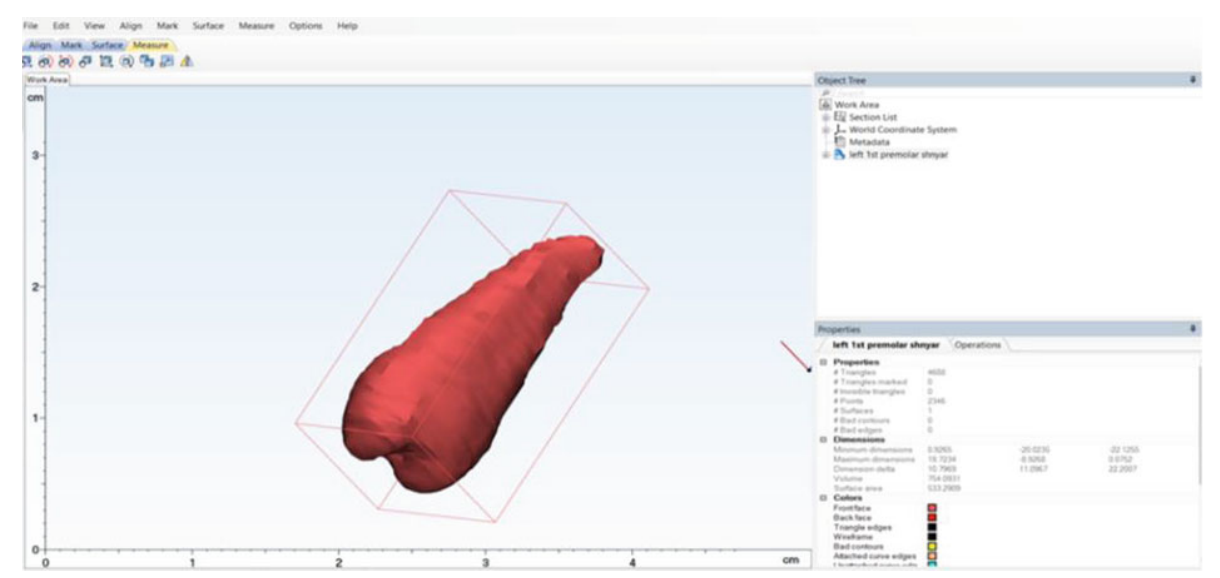

FIGURE 4: 3Matic software for converting the three-dimensional tooth model from DICOM to STL.

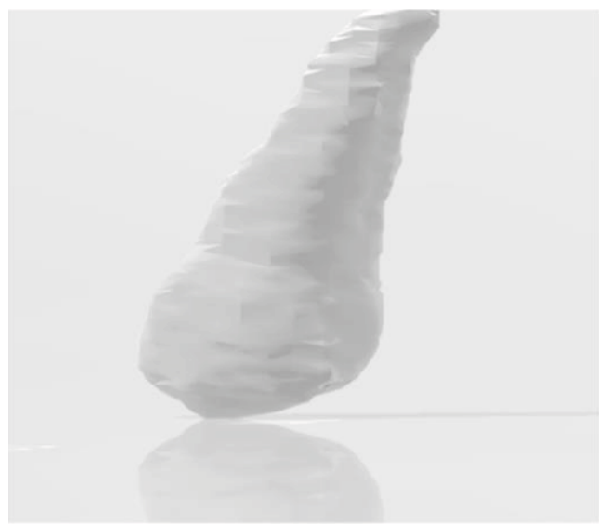

(a)

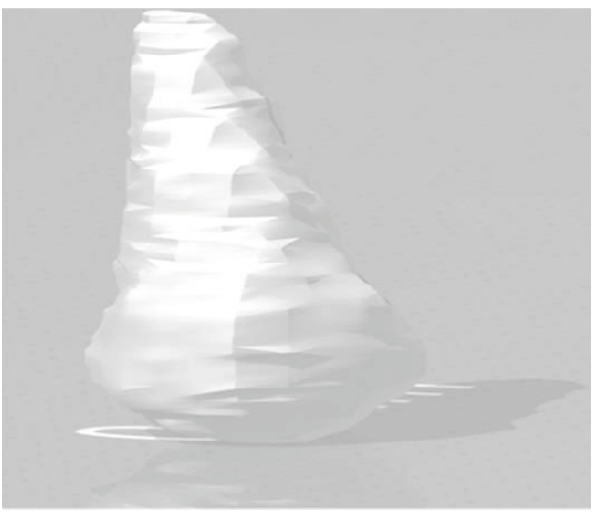

(b)

FiguRE 5: STL photos of the first premolars: (a) fronto-lateral; (b) frontal view of the tooth.

brushed under running water to remove any adherent from the tooth surface (Figure 6), and kept in a labeled container filled with $10 \%$ formalin as a fixative solution.

The physical volume $(\mathrm{PhV})$ of the tooth was measured by the water displacement method [32] in a 5-mL graduated cylinder with gradations of $0.1 \mathrm{~mL}$ (Fisher Scientific, Pittsburgh, Penn). The cylinder was filled with water at room temperature $\left(23.5^{\circ} \mathrm{C}\right)$ to the $4-\mathrm{mL}$ mark. The tooth was cleaned thoroughly and dried with an air syringe then immersed completely in the cylinder, and the new water level was recorded as shown in Figures $7(\mathrm{a})$ and $7(\mathrm{~b}))$. The readings should be taken at the lowest portion of the meniscus.

The volume of the displaced water which determines the $\mathrm{PhV}$ was obtained by subtracting the initial water volume from the final volume after immersing the tooth in the water $[27,28]$. For reducing the bias, the volume of each tooth was measured twice and the average of the two readings was calculated.

2.5. Data Statistical Analysis. The data was analyzed in SPSS advanced statistics (Statistical Package for Social Sciences), version 21 (SPSS Inc., Chicago, IL). The data was initially

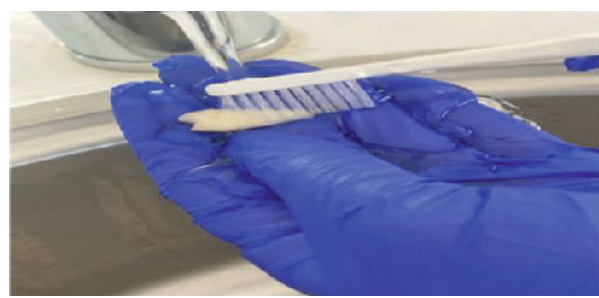

FIgURE 6: Cleaning of the extracted tooth by brush under running water.

examined for normality in distribution using the Kolmogorov-Smirnov test and the Shapiro-Wilk test. The comparisons between the groups for normally distributed numeric variables were done by using the ANOVA test. $\boldsymbol{P}$ $\leq 0.05$ was considered statistically significant.

A comparison between the software and real volume measurements of each tooth was started to check the sensitivity of each program. A correlation within and between groups was performed on the recorded measurements to determine the level of reliability of the data. The $F$ value was obtained for evaluating the statistical significance of the programs. 


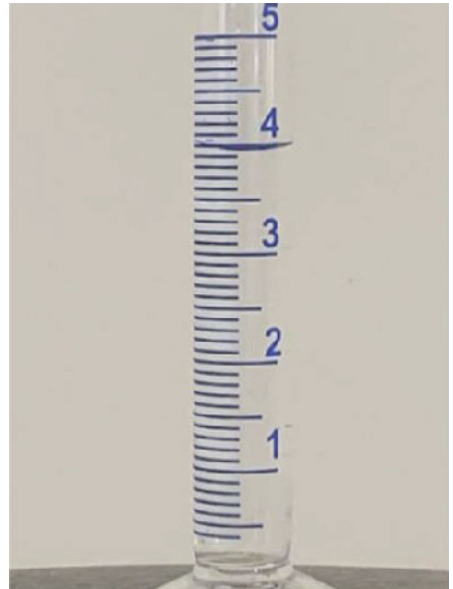

(a)

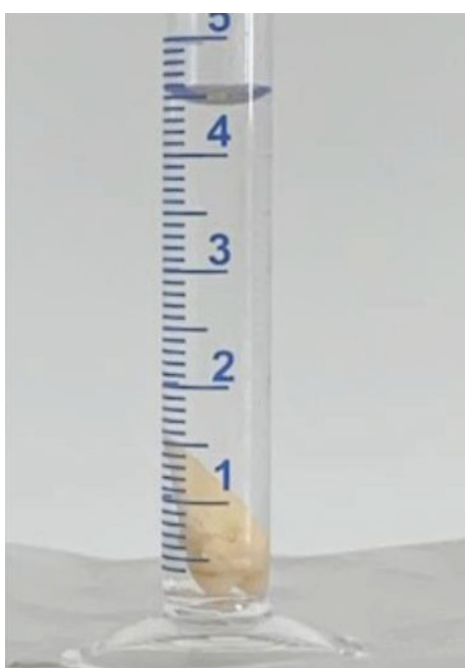

(b)

FIgure 7: Measuring the physical volume of the tooth by water displaced method: (a) before immersing the tooth; (b) tooth inside the cylinder.

\section{Results}

CBCT data can be used to generate 3D printed models that provide accurate lateral cephalograms, visualize growth, help in estimating the age, and evaluate oral and maxillofacial structures which cannot be accurately assessed with traditional 2D radiographs [33-35].

Most of the studies suggested that during the CBCT scanning, increasing the voxel size will increase the volume measurements of the teeth $[21,36,37]$. So, the current study selected the voxel size of $0.150 \mathrm{~mm}$ which is regarded to be within a small range.

The interexaminer calibration between the two observers showed a statistically nonsignificant difference as shown in (Table 1). The overall mean $\mathrm{PhV}$ of the tooth specimens was $0.57592 \mathrm{~mL}$, while the overall mean of VRMesh and 3 Matic tooth volumes was $0.57419 \mathrm{~mL}$ which is equal to $574.19 \mathrm{~mm}^{3}$ for the first evaluation.

The mean value of VRMesh and 3Matic volumes was $0.57850 \mathrm{~mL}$ for the second time evaluation as in Table 2. As observed, the first time evaluated mean values of the software are lower than the $\mathrm{PhV}$ volume, while the second time mean evaluations are greater. But the differences are of no significance from a statistical point of view.

The mean of each observation was compared to the PhV to determine the accuracy of segmentation. There was a statistically nonsignificant difference between the values obtained from CBCT volumes, which were evaluated by the 3Matic and VRMesh software after the segmentation, and $\mathrm{PhV}$. Also, when the groups of different evaluations were analyzed by the ANOVA test, they showed statistically nonsignificant differences (Table 3 ). These results support the accuracy of both software in relation to the real tooth size.

All the values are normally distributed when tested by both Kolmogorov-Smirnov and Shapiro-Wilk as illustrated in Table 4 . When evaluating the volume of the tooth by the
TABLE 1: ANOVA test analysis for comparing the mean by the radiographic volume measurements of two observers.

\begin{tabular}{lccccc}
\hline \multicolumn{5}{c}{ ANOVA test } & \\
Data & $\begin{array}{l}\text { Sum of } \\
\text { squares }\end{array}$ & df & $\begin{array}{c}\text { Mean } \\
\text { square }\end{array}$ & $F$ & Sig. \\
\hline $\begin{array}{l}\text { Between } \\
\text { groups }\end{array}$ & 0.00002 & 3 & 0.000 & 0.00213 & 0.99986 \\
$\begin{array}{l}\text { Within } \\
\text { groups }\end{array}$ & 0.31418 & & 0.00413 & & \\
Total & 0.31421 & & & & \\
\hline
\end{tabular}

df: degree of freedom, Sig.: significance.

3Matic and the VRMesh software, it was noticed that they were almost had the same value, except for some negligible decimals.

In Figure 8, the normal distribution of physical volume measurements that were compared to the expected normal was observed.

Both the CBCT software measurements of the first time were normally distributed (VRMesh 1, 3Matic 1) when compared to the expected normal as illustrated in Figures 9 and 10 .

Figures 11 and 12 explain the normal distribution of the second groups of the CBCT software measurements (VRMesh 2, 3Matic 2) when compared to the expected normal.

\section{Discussion}

The introduction of CBCT imaging into the orthodontic branch has popularized the idea of volumetric analysis for both anatomic visualization and biomechanical considerations [12]. Advances in the CBCT technology make it feasible for this imaging process to be the standard of care in orthodontic practice. However, the realization of its full potential in everyday diagnosis and treatment planning 
TABLE 2: Descriptive data of physical volume and radiographic volume (mL).

\begin{tabular}{|c|c|c|c|c|c|c|}
\hline & & & scriptive stat & & & \\
\hline & $N$ & Minimum & Maximum & & & Std. deviation \\
\hline & Statistic & Statistic & Statistic & Statistic & Std. error & Statistic \\
\hline $\mathrm{PhV}$ & 26 & 0.474 & 0.674 & 0.57592 & 0.011356 & 0.057902 \\
\hline VRMesh1 & 26 & 0.456 & 0.668 & 0.57419 & 0.012207 & 0.062245 \\
\hline 3Matic1 & 26 & 0.456 & 0.668 & 0.57419 & 0.012207 & 0.062245 \\
\hline VRMesh 2 & 26 & 0.462 & 0.690 & 0.57850 & 0.012828 & 0.065409 \\
\hline 3Matic2 & 26 & 0.462 & 0.690 & 0.57850 & 0.012828 & 0.065409 \\
\hline
\end{tabular}

Std.: standard.

TABLE 3: ANOVA test analysis for comparing the mean by physical and radiographic volume measurements.

\begin{tabular}{lccccc}
\hline \multicolumn{5}{c}{ ANOVA test } & \\
Data & Sum of squares & df & $\begin{array}{c}\text { Mean } \\
\text { square }\end{array}$ & $F$ & Sig. \\
\hline $\begin{array}{l}\text { Between } \\
\text { groups }\end{array}$ & 0.000 & 4 & 0.000 & 0.031 & 0.998 \\
Within groups & 0.491 & 125 & 0.004 & & \\
Total & 0.492 & 129 & & & \\
\hline
\end{tabular}

df: degree of freedom; Sig.: significance.

TABLE 4: The normality tests used for the data distribution.

\begin{tabular}{|c|c|c|c|c|c|c|}
\hline & & ests & normal & & & \\
\hline & Kolmog & ov-S & $\operatorname{irnov}^{a}$ & Sha & ro- $W$ & \\
\hline & Statistic & $\mathrm{df}$ & Sig. & Statistic & $\mathrm{df}$ & Sig. \\
\hline $\mathrm{PhV}$ & 0.150 & 26 & 0.136 & 0.945 & 26 & 0.178 \\
\hline VRMesh 1 & 0.137 & 26 & $.200^{*}$ & 0.950 & 26 & 0.236 \\
\hline 3Matic 1 & 0.137 & 26 & $.200^{*}$ & 0.950 & 26 & 0.236 \\
\hline VRMesh 2 & 0.141 & 26 & 0.197 & 0.955 & 26 & 0.298 \\
\hline 3Matic 2 & 0.141 & 26 & 0.197 & 0.955 & 26 & 0.298 \\
\hline
\end{tabular}

requires its validation through accuracy and reliability studies [38].

CBCT imaging has a high degree of measurement accuracy in horizontal, vertical, and angular measurements as well as the panorama and three-dimensional views in the areas of the dentomaxillofacial region [39].

The volumetric analysis of the tooth requires segmentation from the surrounding structures [21, 22]. The MIMICS software has different options for segmentation and has a slight learning curve [40].

Several previous studies have concentrated on the accuracy of the CBCT data in measuring the volume of the teeth $[12,41,42]$. The acceptance of CBCT as a tool for evaluating both root lengths and volumes has been focused on in numerous studies [12, 43-54].

In the current study, semiautomated segmentation was chosen with the entire tooth three-dimensional volume examination instead of only the dental roots apical to the cemento-enamel junction (CEJ) as it is considered to be more definite [30].
Among the various possible uses of segmentation, calculating the volume is of great interest in dentistry. In legal odontology, measuring the volume of a tooth provides access to estimating the individual age [55].

A number of previous studies also performed the in vivo tooth evaluation from CBCT by the segmentation of the tooth from the surrounding structures [12, 19, 56, 57]. The strength and accuracy of CBCT for dental volume measurements in in vivo has been shown statistically not significantly different as in vitro measurements in a study done by Li et al. [54] and even when compared to in vitro micro-CT imaging methods in its accuracy [43].

Some studies like the current study compared the physical volume of the tooth to the volume obtained from the CBCT [12]. The purpose of the current study was to test the accuracy of the volume measurements derived from CBCT images by two different software. In this study, the CBCT scan tooth volume measured by two different software was compared to physical real volume measurements, and the selected teeth extracted were the upper first premolars. To the extent of our knowledge, it was the first time the VRMesh and 3Matic (from MIMICS) software were used to evaluate the in vivo volumetric determinations of the teeth from CBCT.

However, in the current study, several factors significantly influenced the accuracy of the CBCT scan model: the Hounsfield unit threshold settings of segmentation, voxel size, artifacts, tube current, tube voltage, fields of view, and the surrounding tissues [12, 41, 58-62].

The interexaminer calibrations by the ANOVA test revealed statistically nonsignificant differences which indicates the reliability of the observers in the segmentation procedure. The result agreed with Liu et al. [12] and Fadili et al. [29].

In this study, the results showed a nonsignificant difference between the VRMesh and 3Matic groups and the physical volume group. These findings mean that both software can be dependable in the in vivo measurements if the segmentation method was done in a qualified way. This showed a disagreement with Liu et al. [12] who found statistically significant differences between the physical volume of the extracted tooth and the volume obtained from the CBCT images after segmentation, and the authors believed that the quality of CBCT is an important factor for the extraction accuracy. 


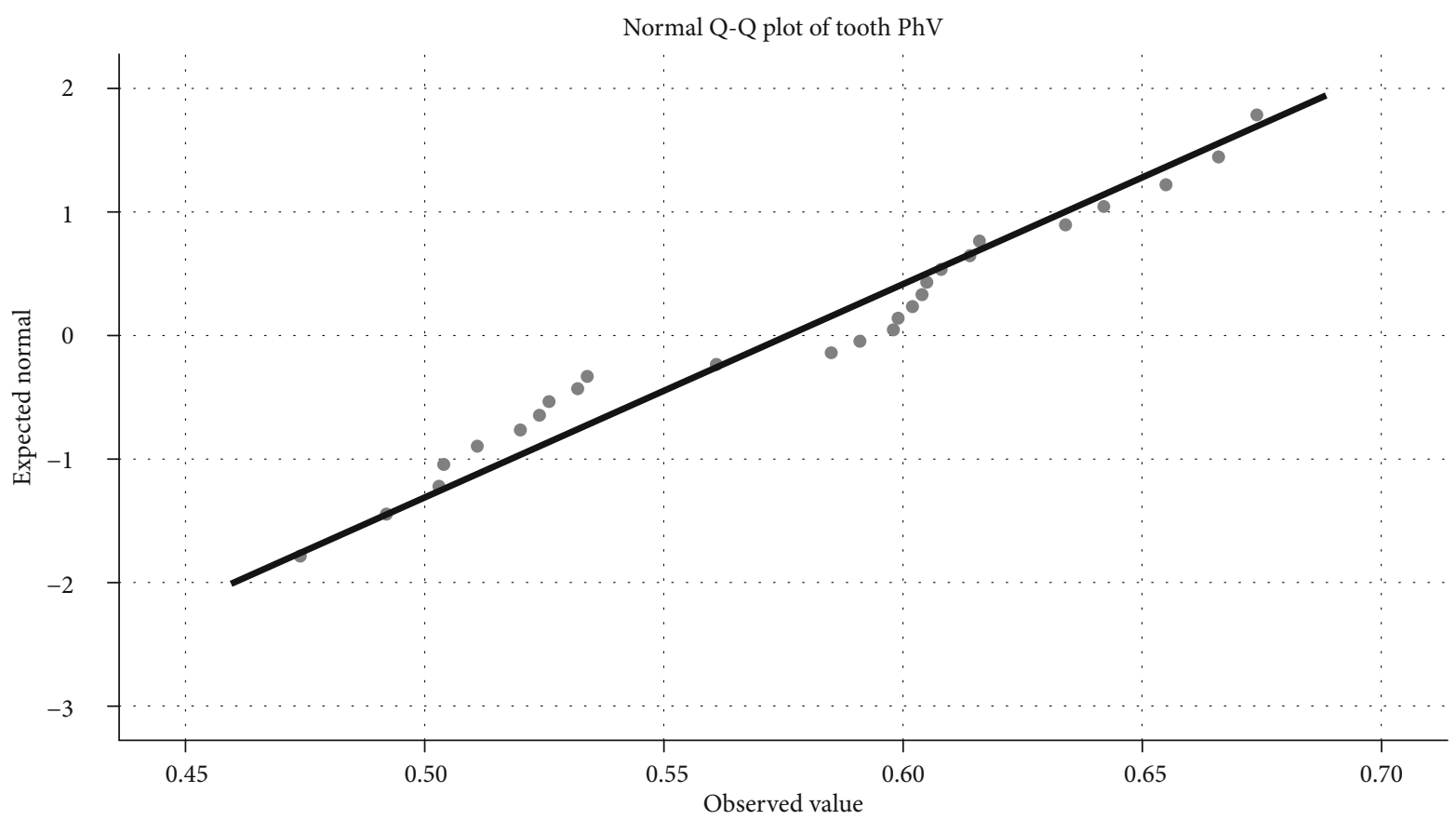

FIGURE 8: Normal distribution of physical volume measurements.

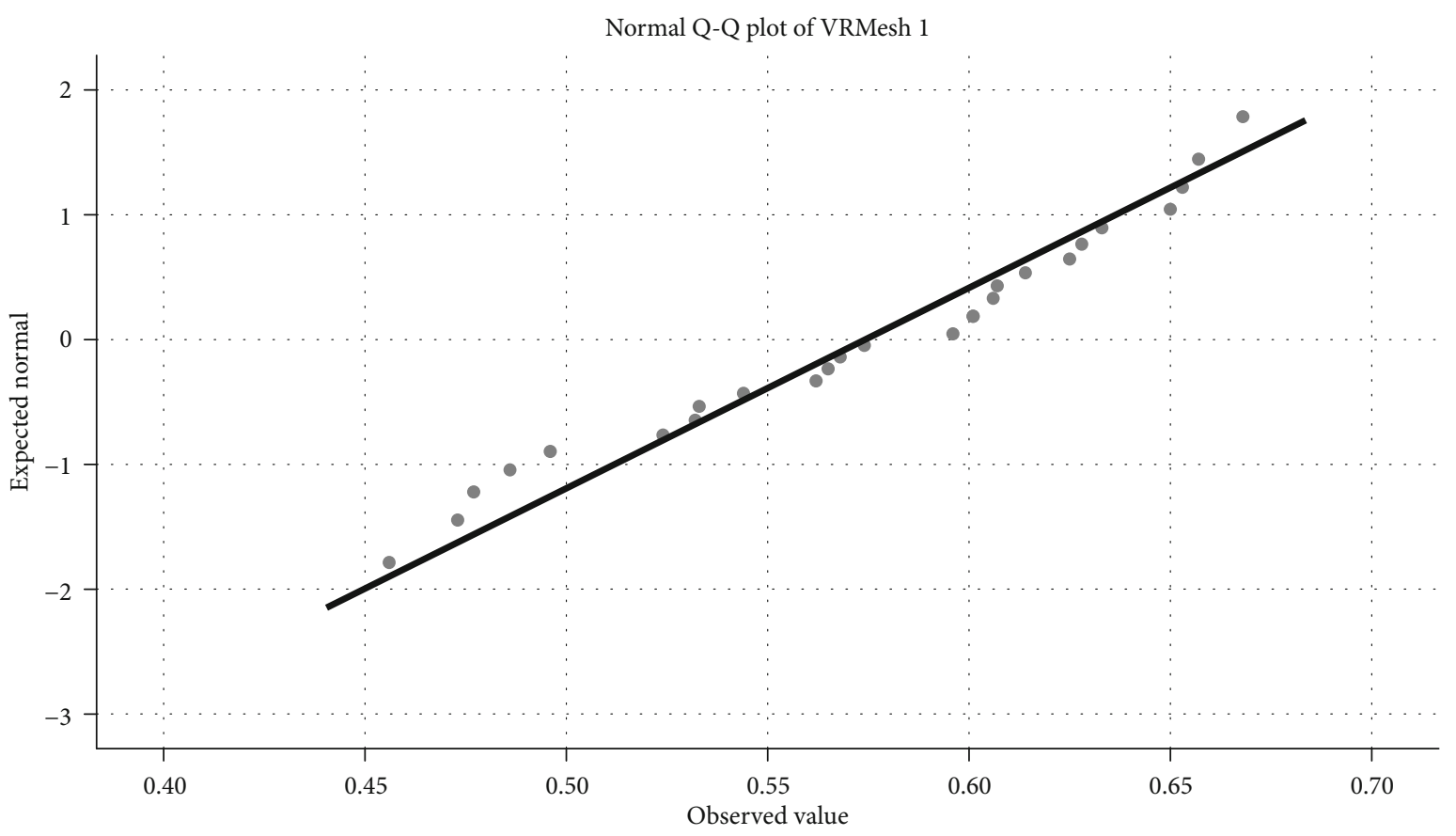

FIGURE 9: Normal distribution of VRMesh 1 volume measurements.

In contrast to the present study, Wang et al. [63] revealed a statistically significant difference in in vivo bucco-lingual, mesio-distal, and root length dimensions between the reference and test models. They suggest that the possible reason for the difference may be due to the differences in the scanning accuracy of CBCT which was used in the test group and the Smart Optics system they used in the reference group.
The nonsignificant difference in this study may be due to the fact that a more accurate segmentation technique was applied in the current study through the use of a mouse pen as a tool for the slice-by-slice refinement, which increased the control during the outlining of the tooth, while the result of the present study agreed with Sang et al. [22] as they found the reconstructed $3 \mathrm{D}$ tooth model from CBCT data can obtain a high linear, volumetric, and geometric accuracy. 


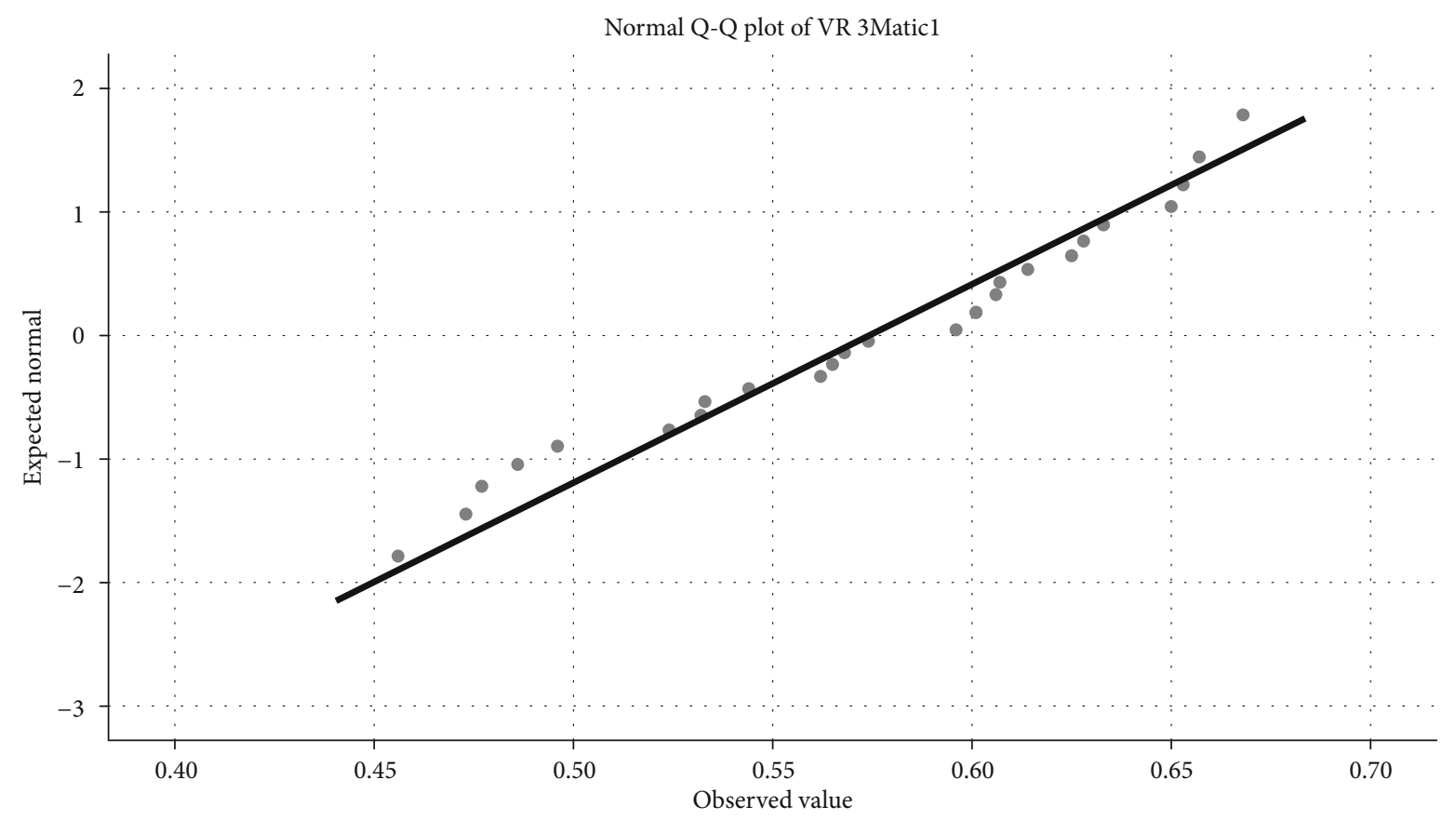

Figure 10: Normal distribution of 3Matic 1volume measurements.

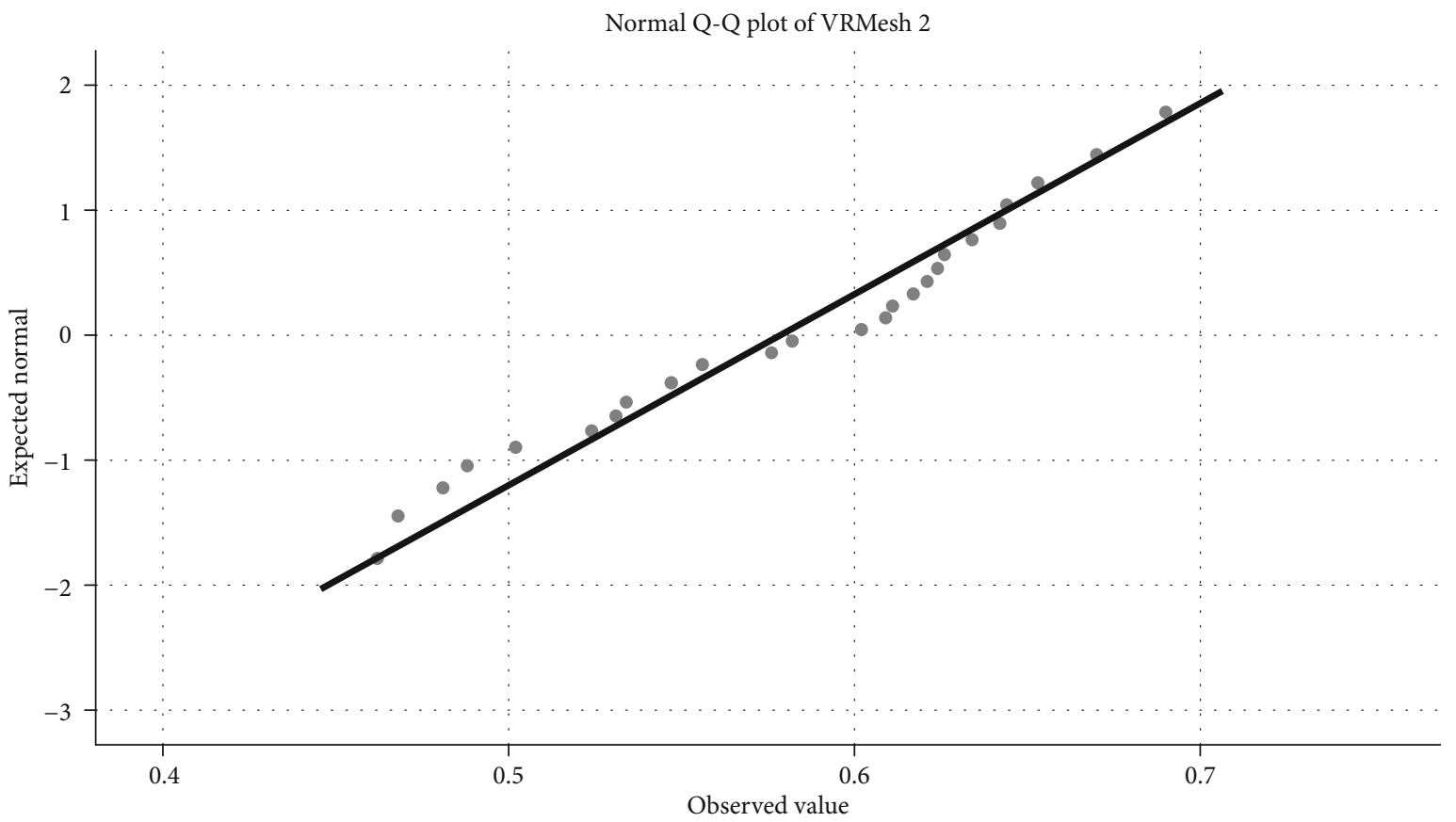

FIGURE 11: Normal distribution of VRMesh 2 volume measurements.

Generally, when voxel sizes are constant, it seems that the changes of software or CBCT machine have no significant clinical importance due to the fact that all the types of 3D software programs are used as a tool to calculate a volume obtained from the CBCT.

In recent years, several studies have focused on the linear and volumetric accuracy measurements of $\mathrm{CBCT}$ images, but the results are controversial. In studies done by Maret et al.
[41] and Wang et al. [42], they found that the CBCT volume measurements of the teeth were similar to those with tomography scan parameters. Although Liu et al. [12] even support the feasibility of in vivo dental volume measurements by using $\mathrm{CBCT}$ [12], they suggested that the $\mathrm{CBCT}$ volume measurements differed slightly from the physical volumes, by $-4 \%$ to $7 \%$. In the current study, the deviation of tooth volumes may be due to the segmentation procedure and/or 


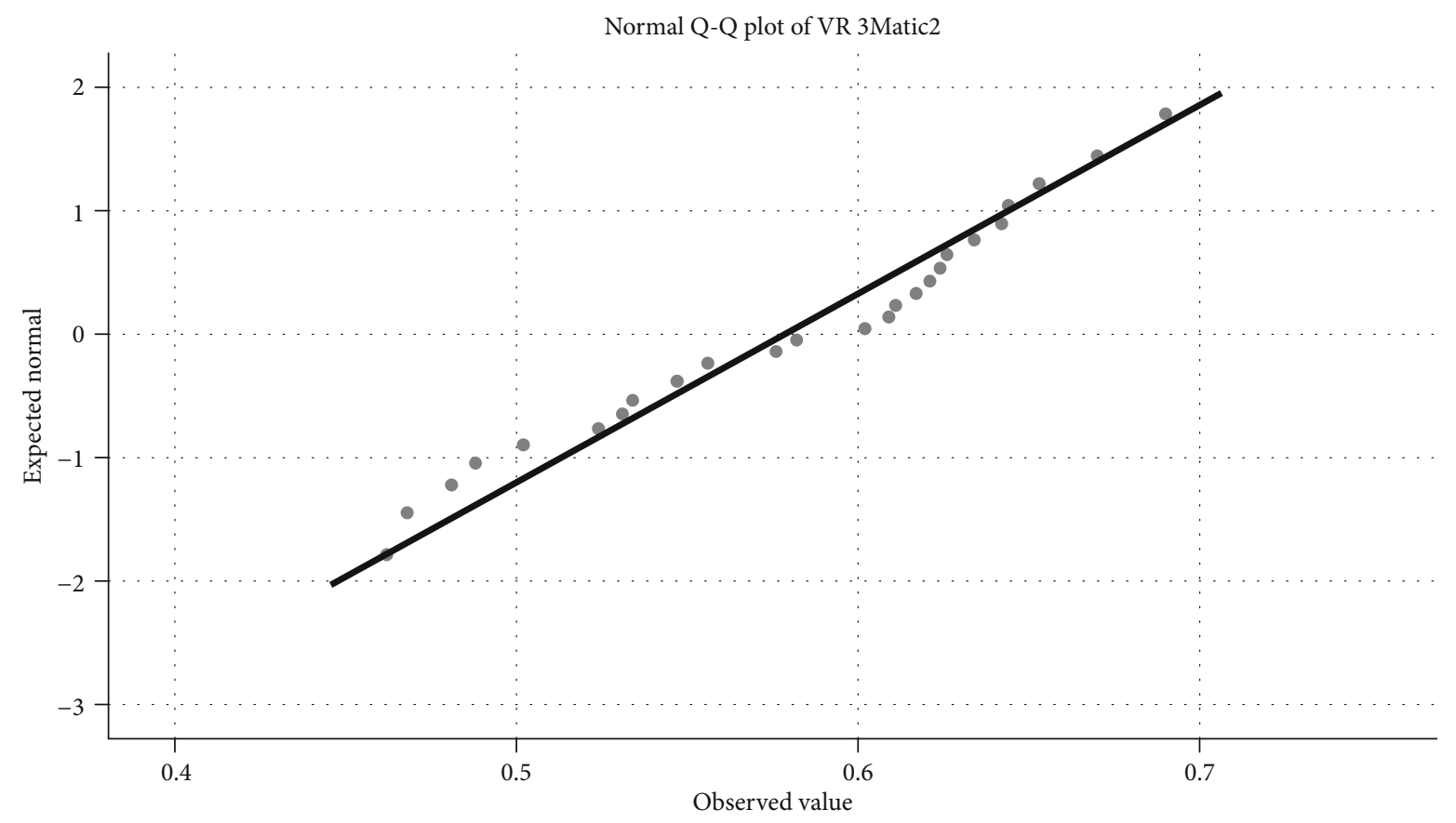

FIGURE 12: Normal distribution of 3Matic 2 volume measurements.

the Hounsfield unit threshold value setting during segregation. In addition, voxel sizes, tube voltages, and fields of view from the same CBCT device could also affect tooth volumes.

In the current study, the mean physical volumes obtained through PhV measurements were larger by $0.00173 \mathrm{~mL}$ than the mean volumetric measurements by CBCT. On the contrary, in the second round of calculations, the mean volumetric measurements by CBCT were generally larger by $0.00258 \mathrm{~mL}$ than the mean physical volumes obtained through $\mathrm{PhV}$ measurements. This difference in the mean suggests that the subjective aspects of segmentation and any false during the manual slice-by-slice refinement procedure may affect the volumetric measurements. Because the differences were so little, they are regarded as clinically nonsignificant. So, in agreement with Liu et al. [12], the in vivo tooth volume determination from CBCT data is practicable.

A common use of segmented tooth models in orthodontics is to conduct various study model analyses, such as arch length discrepancy and Bolton analysis. These data suggest that the differences in segmentation are relatively small and would not likely influence common study model analysis for the diagnosis and treatment planning.

There are many factors that could affect the accuracy of segmentation, such as image quality, which is highly predominant in segmentation. CBCT imaging quality can be related to machine settings, patient positioning and management, volume reconstruction, and DICOM export. So, if all these factors are set in a good manner, the results will be more dependable in the diagnosis and treatment planning.

The results of the current study are in agreement with $\mathrm{Li}$ et al. [54] who revealed a statistically nonsignificant difference in the accuracy between the in vivo measurements obtained from the CBCT and in vitro measurements from laser scanning.
Nimbalkar [40]concluded that the volume of the teeth depends on the threshold interval and the segmentation methods, which differed for each software and operator. All the software packages used different segmentation tools, and there is no fixed protocol or algorithm for preparing the DICOM images for the assessment of the volume of teeth, and there are various methods for volume assessment that are commercially available.

\section{Conclusion}

The assessment of in vivo tooth volume measurement with different three-dimensional imaging software (VRMesh and 3 Matic programs) in comparison with the tooth physical volume is reliable. The use of a mouse pen as a tool during the refining stage of semiautomated segmentation may have helped in decreasing the errors and increasing the accuracy of the procedure. With advancements in the technology and the CBCT devices, the in vivo volumetric determinations of the teeth are dependable and can be applied in orthodontic diagnosis and treatment planning.

\section{Data Availability}

All the data supporting the results can be found under request through the corresponder's email at any time.

\section{Conflicts of Interest}

The authors declare that there are no conflicts of interest regarding the publication of this article. 


\section{References}

[1] P. Mozzo, C. Procacci, A. Tacconi, P. Tinazzi Martini, and I. A. Bergamo Andreis, "A new volumetric CT machine for dental imaging based on the cone-beam technique: preliminary results," European Radiology, vol. 8, no. 9, pp. 1558-1564, 1998.

[2] D. C. Hatcher, "Operational principles for cone-beam computed tomography," The Journal of the American Dental Association, vol. 141, no. 3, pp. 3S-6S, 2010.

[3] S. Kapila, R. S. Conley, and W. E. Harrell Jr., "The current status of cone beam computed tomography imaging in orthodontics," Dento maxillofacial Radiology, vol. 40, no. 1, pp. 2434, 2011.

[4] S. D. Kapila and J. M. Nervina, "CBCT in orthodontics: assessment of treatment outcomes and indications for its use," Dentomaxillofacial Radiology, vol. 44, no. 1, 2015.

[5] D. Wiechmann, V. Rummel, A. Thalheim, J. S. Simon, and L. Wiechmann, "Customized brackets and archwires for lingual orthodontic treatment," American Journal of Orthodontics and Dentofacial Orthopedics, vol. 124, no. 5, pp. 593599, 2003.

[6] M. Robiony, I. Salvo, F. Costa et al., "Accuracy of virtual reality and stereolithographic models in maxillo-facial surgical planning," Journal of Craniofacial Surgery, vol. 19, no. 2, pp. 482-489, 2008.

[7] F. Garino and G. B. Garino, "Digital treatment objectives: procedure and clinical application," Progress in Orthodontics, vol. 5, pp. 248-256, 2004.

[8] W. C. Scarfe and A. G. Farman, Cone-beam computed tomography oral radiology: principles and interpretation, Mosby Elsevier, St. Louis, MO, 6th edition, 2009.

[9] W. C. Scarfe and A. G. Farman, "What is cone-beam CT and how does it work?," Dental Clinics of North America, vol. 52, no. 4, pp. 707-730, 2008.

[10] A. G. Farman and W. C. Scarfe, "The basics of maxillofacial cone beam computed tomography," Seminars in Orthodontics, vol. 15, no. 1, pp. 2-13, 2009.

[11] J. Mah and D. Hatcher, "Three-dimensional craniofacial imaging," American Journal of Orthodontics and Dentofacial Orthopedics, vol. 126, no. 3, pp. 308-309, 2004.

[12] Y. Liu, R. Olszewski, E. S. Alexandroni, R. Enciso, T. Xu, and J. K. Mah, "The validity of in vivo tooth volume determinations from cone-beam computed tomography," The Angle Orthodontist, vol. 80, no. 1, pp. 160-166, 2010.

[13] M. O. Lagravère, J. Carey, R. W. Toogood, and P. W. Major, "Three-dimensional accuracy of measurements made with software on cone-beam computed tomography images," American Journal of Orthodontics and Dentofacial Orthopedics, vol. 134, no. 1, pp. 112-116, 2008.

[14] E. Venkatesh and S. V. Elluru, "Cone beam computed tomography: basics and applications in dentistry," Journal of Istanbul University Faculty of Dentistry, vol. 51, 3 Supplement 1, pp. S102-S121, 2017.

[15] R. Pauwels, O. Nackaerts, N. Bellaiche et al., "Variability of dental cone beam CT grey values for density estimations," The British Journal of Radiology, vol. 86, no. 1021, 2013.

[16] A. Macchi, G. Carrafiello, V. Cacciafesta, and A. Norcini, "Three-dimensional digital modeling and setup," American Journal of Orthodontics and Dentofacial Orthopedics, vol. 129, no. 5, pp. 605-610, 2006.
[17] F. Garino and G. Garino, "Computer-aided interactive indirect bonding," Progress in Orthodontics, vol. 6, no. 2, pp. 214-223, 2005.

[18] F. Toso, C. Zuiani, M. Vergendo et al., "Usefulness of computed tomography in pre-surgical evaluation of maxillo-facial pathology with rapid prototyping and surgical pre-planning by virtual reality," Radiologia Medica, vol. 110, pp. 665-675, 2005.

[19] D. R. Periago, W. C. Scarfe, M. Moshiri, J. P. Scheetz, A. M. Silveira, and A. G. Farman, "Linear accuracy and reliability of cone beam CT derived 3-dimensional images constructed using an orthodontic volumetric rendering program," The Angle Orthodontist, vol. 78, no. 3, pp. 387-395, 2008.

[20] S. A. Stratemann, J. C. Huang, K. Maki, D. C. Hatcher, and A. J. Miller, "Evaluating the mandible with cone-beam computed tomography," American Journal of Orthodontics and Dentofacial Orthopedics, vol. 137, no. 4, pp. S58-S70, 2010.

[21] N. Ye, F. Jian, J. Xue et al., "Accuracy of in-vitro tooth volumetric measurements from cone-beam computed tomography," American Journal of Orthodontics and Dentofacial Orthopedics, vol. 142, no. 6, pp. 879-887, 2012.

[22] Y. H. Sang, H. C. Hu, S. H. Lu, Y. W. Wu, W. R. Li, and Z. H. Tang, "Accuracy assessment of three-dimensional surface reconstructions of in vivo teeth from cone-beam computed tomography," Chinese Medical Journal, vol. 129, no. 12, pp. 1464-1470, 2016.

[23] K. Bhargavi and S. Jyothi, "A survey on threshold based segmentation technique in image processing," International Journal of Innovative Research Development, vol. 3, pp. 234239, 2014.

[24] D. Grauer, L. S. H. Cevidanes, and W. R. Proffit, "Working with DICOM craniofacial images," American Journal of Orthodontics and Dentofacial Orthopedics, vol. 136, no. 3, pp. 460-470, 2009.

[25] E. Shaheen, W. Khalil, M. Ezeldeen et al., "Accuracy of segmentation of tooth structures using 3 different CBCT machines," Oral Surgery, Oral Medicine, Oral Pathology and Oral Radiology, vol. 123, no. 1, pp. 123-128, 2017.

[26] N. P. Birur, S. Patrick, K. Gurushanth, A. S. Raghavan, and S. Gurudath, "Comparison of gray values of cone-beam computed tomography with hounsfield units of multislice computed tomography: an in vitro study," Indian Journal of Dental Research, vol. 28, no. 1, pp. 66-70, 2017.

[27] G. Forbes, D. G. Gehring, C. A. Gorman, M. D. Brennan, and I. T. Jackson, "Volume measurements of normal orbital structures by computed tomographic analysis," American Journal of Roentgenology, vol. 145, no. 1, pp. 149-154, 1985.

[28] J. Jensen, J. Kragskov, A. Wenzel, and S. Sindet-Pedersen, "In vitro analysis of the accuracy of subtraction radiography and computed tomography scanning for determination of bone graft volume," Journal of Oral and Maxillofacial Surgery, vol. 56, no. 6, pp. 743-748, 1998.

[29] A. Fadili, A. Halimi, H. Benyahia, and F. Zaoui, "Stereology volume analysis to evaluate teeth's root using CBCT images," Reports in Medical Imaging, vol. 11, pp. 31-39, 2018.

[30] D. Forst, S. Nijjar, C. Flores-Mir, J. Carey, M. Secanell, and M. Lagravere, "Comparison of in vivo 3D cone-beam computed tomography tooth volume measurement protocols," Progress in Orthodontics, vol. 15, no. 1, p. 69, 2014.

[31] Z. Yang, L. Fan, K. Kwon et al., “Age estimation for children and young adults by volumetric analysis of upper anterior teeth using CBCT data," Folia Morphologica, 2015. 
[32] S. A. Stratemann, J. C. Huang, K. Maki, A. J. Miller, and D. C. Hatcher, "Comparison of cone beam computed tomography imaging with physical measures," Dentomaxillofacial Radiology, vol. 37, no. 2, pp. 80-93, 2008.

[33] A. Ghoneima, E. Allam, K. Kula, and L. Jack, "Three-dimensional imaging and software advances in orthodontics," in Orthodontics - Basic Aspects and Clinical Considerations, Farid Bourzgui, IntechOpen, 2012.

[34] V. Kumar, J. Ludlow, A. Mol, and L. Cevidanes, "Comparison of conventional and cone beam CT synthesized cephalograms," Dento Maxillo Facial Radiology, vol. 36, no. 5, pp. 263-269, 2007.

[35] J. Montúfar, M. Romero, V. Muñoz-Jiménez, R. ScougallVilchis, and B. Jiménez, "Perspective and orthogonal $\mathrm{CBCT} / \mathrm{CT}$ digitally reconstructed radiographs compared to conventional cephalograms," Conf. Biomedical Engineering and Sciences|BIOENG'16|, pp. 41-45, 2018.

[36] J. K. Mah, L. Yi, R. C. Huang, and H. Choo, "Advanced applications of cone beam computed tomography in orthodontics," Seminars in Orthodontics, vol. 17, no. 1, pp. 57-71, 2011.

[37] A. H. Jheon, S. Oberoi, R. C. Solem, and S. Kapila, "Moving towards precision orthodontics: an evolving paradigm shift in the planning and delivery of customized orthodontic therapy," Orthodontics \& Craniofacial Research, vol. 20, pp. 106-113, 2017.

[38] K. Adarsh, P. Sharma, and A. Juneja, "Accuracy and reliability of tooth length measurements on conventional and CBCT images: an in vitro comparative study," Journal of Orthodontic Science, vol. 7, no. 1, p. 17, 2018.

[39] M. Abdinian and H. Baninajarian, "The accuracy of linear and angular measurements in the different regions of the jaw in cone-beam computed tomography views," Dental Hypotheses, vol. 8, no. 4, p. 100, 2017.

[40] S. Nimbalkar, Accuracy of volumetric analysis software packages in assessment of tooth volume using CBCT, vol. 400, Loma Linda University Electronic Theses, Dissertations \& Projects, 2016, https://scholarsrepository.llu.edu/etd/400/.

[41] D. Maret, F. Molinier, J. Braga et al., "Accuracy of 3D reconstructions based on cone beam computed tomography," Journal of Dental Research, vol. 89, no. 12, pp. 1465-1469, 2010.

[42] Y. Wang, S. He, L. Yu, J. Li, and S. Chen, "Accuracy of volumetric measurement of teeth in vivo based on cone beam computer tomography," Orthodontics and Craniofacial Research, vol. 14, no. 4, pp. 206-212, 2011.

[43] S. N. Ponder, E. Benavides, S. Kapila, and N. E. Hatch, "Quantification of external root resorption by low- vs highresolution cone-beam computed tomography and periapical radiography: a volumetric and linear analysis," American Journal of Orthodontics and Dentofacial Orthopedics, vol. 143, no. 1, pp. 77-91, 2013.

[44] S. Ericson and J. Kurol, "Incisor root resorptions due to ectopic maxillary canines imaged by computerized tomography: a comparative study in extracted teeth," The Angle Orthodontist, vol. 70, no. 4, pp. 276-283, 2000.

[45] H. Lund, K. Grondahl, and H. G. Grondahl, "Cone beam computed tomography for assessment of root length and marginal bone level during orthodontic treatment," The Angle Orthodontist, vol. 80, no. 3, pp. 466-473, 2010.

[46] S. Y. Kim, S. H. Lim, S. N. Gang, and H. J. Kim, "Crown and root lengths of incisors, canines, and premolars measured by cone-beam computed tomography in patients with malocclusions," Korean Journal of Orthodontics, vol. 43, no. 6, pp. 271-278, 2013.

[47] Y. Wang, S. He, Y. Guo, S. Wang, and S. Chen, "Accuracy of volumetric measurement of simulated root resorption lacunas based on cone beam computed tomography," Orthodontics and Craniofacial Research, vol. 16, no. 3, pp. 169-176, 2013.

[48] Z. Dalili, M. Taramsari, S. Z. Mousavi Mehr, and F. Salamat, "Diagnostic value of two modes of cone-beam computed tomography in evaluation of simulated external root resorption: an in vitro study," Imaging Science in Dentistry, vol. 42, no. 1, pp. 19-24, 2012.

[49] A. Shokri, H. Mortazavi, F. Salemi, A. Javadian, H. Bakhtiari, and H. Matlabi, "Diagnosis of simulated external root resorption using conventional intraoral film radiography, CCD, PSP, and CBCT: a comparison study," Biomedical Journal, vol. 36, no. 1, pp. 18-22, 2013.

[50] H. Ren, J. Chen, F. Deng, L. Zheng, X. Liu, and Y. Dong, "Comparison of cone-beam computed tomography and periapical radiography for detecting simulated apical root resorption," The Angle Orthodontist, vol. 83, no. 2, pp. 189-195, 2013.

[51] I. O. Castro, A. H. G. Alencar, J. Valladares-Neto, and C. Estrela, "Apical root resorption due to orthodontic treatment detected by cone beam computed tomography," The Angle Orthodontist, vol. 83, no. 2, pp. 196-203, 2013.

[52] X. Y. Xie and Z. Y. Zhang, "Diagnostic accuracy of cone beam computed tomography and eight-slice computed tomography for evaluation of external root reabsorption," Beijing Da Xue Xue Bao. Journal of Peking University. Health Science, vol. 44, no. 4, pp. 628-632, 2012.

[53] H. Lund, K. Grondahl, K. Hansen, and H. G. Grondahl, "Apical root resorption during orthodontic treatment," The Angle Orthodontist, vol. 82, no. 3, pp. 480-487, 2012.

[54] W. Li, F. Chen, F. Zhang et al., "Volumetric measurement of root resorption following molar mini-screw implant intrusion using cone beam computed tomography," PLoS One, vol. 8, no. 4, article e60962, 2013.

[55] T. Y. Marroquin, S. Karkhanis, S. I. Kvaal, S. Vasudavan, E. Kruger, and M. Tennant, "Age estimation in adults by dental imaging assessment systematic review," Forensic Science International, vol. 275, pp. 203-211, 2017.

[56] A. Baysal, I. Karadede, S. Hekimoglu et al., "Evaluation of root resorption following rapid maxillary expansion using conebeam computed tomography," The Angle Orthodontist, vol. 82, no. 3, pp. 488-494, 2012.

[57] J.-H. Yu, K.-W. Shu, M.-T. Tsai, J.-T. Hsu, H.-W. Chang, and K.-L. Tung, "A cone-beam computed tomography study of orthodontic apical root resorption," Journal of Dental Sciences, vol. 8, no. 1, pp. 74-79, 2013.

[58] Z. Fourie, J. Damstra, R. H. Schepers, P. O. Gerrits, and Y. Ren, "Segmentation process significantly influences the accuracy of 3D surface models derived from cone beam computed tomography," European Journal of Radiology, vol. 81, no. 4, pp. e524e530, 2012.

[59] J. Damstra, Z. Fourie, J. J. R. Huddleston Slater, and Y. Ren, "Accuracy of linear measurements from cone-beam computed tomography-derived surface models of different voxel sizes," American Journal of Orthodontics and Dentofacial Orthopedics, vol. 137, no. 1, pp. 16.e1-16.e6, 2010. 
[60] X. Ma and Z. Zhang, Clinical Application of Oral and Maxillofacial Cone Beam Computed Tomography, People's Medical Publishing House, Beijing, China, 2011.

[61] J. Sur, K. Seki, H. Koizumi, K. Nakajima, and T. Okano, "Effects of tube current on cone-beam computerized tomography image quality for presurgical implant planning in vitro," Oral Surgery, Oral Medicine, Oral Pathology, Oral Radiology, and Endodontology, vol. 110, no. 3, pp. e29-e33, 2010.

[62] R. Schulze, U. Heil, D. Gross et al., "Artefacts in CBCT: a review," Dentomaxillofacial Radiology, vol. 40, no. 5, pp. 265-273, 2011.

[63] C. Wang, Y. Liu, S. Wang, Y. Wang, and Y. Zhao, "Evaluation of in vivo digital root reconstruction based on anatomical characteristics of the periodontal ligament using cone beam computed tomography," Scientific Reports, vol. 8, no. 1, p. $269,2018$. 